\title{
Stellar Evolution with Enriched Surface Convection Zones I. General Effects of Planet Consumption
}

\author{
Ann Marie Cody ${ }^{1,2} \&$ Dimitar D. Sasselov ${ }^{1}$
}

\begin{abstract}
Abundance analyses of stars with planets have revealed that their metallicities are enhanced relative to field stars. Such a trend was originally suggested to be due to accretion of iron-rich planetary material. Based on this assumption, we have developed a stellar evolution code to model stars with non-uniform metallicity distributions. We have calculated "polluted" stellar evolution tracks for stars with $\mathrm{M}=0.9-1.2 \mathrm{M}_{\odot}$. Our models encompass a range of initial metal content from $\mathrm{Z}=0.01$ to 0.03 , and include metallicity enhancements within the stellar convection zone corresponding to $\Delta \mathrm{Z}=0.005-0.03$. We find that the primary effects of metal enhancement on stellar structure and evolution are expansion of the convection zone and downward shift of effective temperature. In addition, we have computed the surface metallicities expected for stars of different mass for fixed quantities of pollution; there appears to be no correlation with present observational data on the metallicities of stars known to harbor planets.
\end{abstract}

Subject headings: planetary systems — stars: abundances — stars: chemically peculiar — stars:evolution — stars:individual(HD 209458)

\section{Introduction}

\subsection{Metallicity Trends in Extrasolar Planet Host Stars}

Since the initial discovery of extrasolar planets in 1995 (Mayor \& Queloz 1995), the study of these objects has grown at a rapid rate. Thanks in part to improving Doppler technology and a number of new planet detection techniques, more than 100 extrasolar

\footnotetext{
${ }^{1}$ Dept. of Astronomy, Harvard University, 60 Garden St., Cambridge MA 02138; dsasselov@cfa.harvard.edu

${ }^{2}$ Current address: California Institute of Technology, 1200 East California Boulevard, Pasadena, CA 91125; amc@astro.caltech.edu
} 
planets are known, including several that orbit together in systems around a common star. But with the wealth of data have come new puzzles, one of the most intriguing of which is an emerging connection between stellar metallicity and the presence of planets. Numerous spectroscopic observations have revealed that metallicities of stars with planets (SWPs) are enhanced on average, as compared to abundances in F-G type field stars. Quite a few of the stars now known to be orbited by planets have unusually high metallicities (Gonzalez, Wallerstein \& Saar 1999; Castro et al. 1997). Moreover, Gonzalez (1998) noted that the overall metallicity distribution is shifted relative to that of unbiased solar neighborhood star samples. Further discussion of this offset has been offered by Gonzalez et al. (2001), and Santos, Israelian \& Mayor (2001). The latter find that the mean metallicities for the two different groups are different by 0.25 dex in $[\mathrm{Fe} / \mathrm{H}]$, and Murray \& Chaboyer (2002) report similar results. The most recent study has been carried out by Gonzalez (2003) on a set of 69 SWPs together with 43 field stars from Santos et al. (2001) and Fischer \& Valenti (2003); they confirm the $\sim 0.2$ dex significant offset between the SWP and field star $[\mathrm{Fe} / \mathrm{H}]$ distributions is seen. Statistical analysis has enabled Santos et al. (2001) to determine a probability of $10^{-7}$ that the two distributions are the same. Despite the convincing results, there is some concern that biases in SWP detection methods are responsible for the appearance that SWPs are more metal rich than average. It is well known that the current Doppler searches favor identification of massive planets in small orbits, but reasons for a possible association between close-in orbits and high-metallicity parent stars are not clear. Nevertheless, several analyses (Murray \& Chaboyer 2002; Gonzalez 2003) have addressed these issues and concluded that the combination of potential biases still cannot generate the observed metallicity differences. Because the number of known extrasolar planets is now large enough to be statistically significant, the pattern of excess metallicity in host stars is fairly robust.

With biases for the most part ruled out, several competing theories have been devised to explain the SWP metallicity enhancement trend. The main debate centers on whether this pattern is primordial in origin. If so, one hypothesis maintains that planets preferentially form around stars where there is ample heavy-element material. Hence, those systems that contain above average metallicities will be more likely to spawn planets. It is reasonable that the presence of metallic or rocky material in a circumstellar disk might be favorable for planet formation; Pollack et al. (1996) and Lissauer \& Stewart (1993) have outlined some of the effects of disk composition on this process. Alternatively, some authors have suggested that above-average metal abundances are not primordial, but rather, they are due to the more dynamic phenomenon of planet or planetesimal accretion (Laughlin 2000; Gonzalez et al. 2001). Accumulation of rocky material in the outer layers of a star could produce an observable increase in surface metallicity. In the absence of additional considerations, there is at present no a priori reason to favor one hypothesis over the other. However, it is 
certain that an adequate explanation of the planet/host-star metallicity trend will be crucial to understanding the conditions under which planetary systems form and persist.

\subsection{The Possibility of Planetary Migration and Accretion}

The possibility that stellar abundances may be altered by the addition of infalling rocky planet material is tantalizing from an observational standpoint. Because this sort of enrichment is the result of a dynamical process, as opposed to a preexisting condition, its signature should be readily detectable in the surface abundances of stars, given the proper diagnostics. Nevertheless, any feasible theory of metal accretion must first answer the question of how such material could be delivered to the stellar surface. Perhaps not coincidentally, some of the most metal enriched systems exhibit strong evidence for orbital migration, in which giant planets are forced inward toward their parent stars and settle into short-period orbits. Yet, the idea of migration has taken considerable effort to develop since it does not fit the "established" picture provided by our own solar system, where gas giants lie at substantial distances from the sun, and large-scale inward orbital migration has largely been ruled out (Hahn \& Malhotra 1999).

Orbital migration due to circumstellar disk interaction is one explanation for the puzzling "pile-up" of planets at short distances from their host stars (Lin 1997; Laughlin \& Adams 1997; Trilling et al. 1998). Most stars harboring planets are assumed to be surrounded by protoplanetary disks until ages of 1-10 Myr (Laughlin \& Adams 1997). Before the time of dissipation, tidal forces between a planet and the disk material in which it orbits may be responsible for pushing the planet toward the central star. Gonzalez (1997) linked this idea to the observed metal enhancements in stars with close-in planets. Citing Lin, Bodenheimer \& Richardson's (1996) theory of planet-disk interaction, he hypothesized that high stellar metallicities are directly related to planet migration, in that metal-rich dust ahead of the planet is driven into the star. Other authors have similarly proposed that accretion of material is likely, on the grounds that the amount of time required for a planet to migrate inward is close to the viscous evolution time of the disk (Laughlin \& Adams 1997).

Even if pollution is not the main explanation for the high metallicities of SWPs, more direct evidence for engulfment of planets has been reported in stellar abundance anomalies. Israelian et al. (2001, 2003) suggest that the presence of ${ }^{6} \mathrm{Li}$ in stellar atmospheres is an excellent tracer of rocky material accretion, since this isotope should not usually be present in stars whose convection zones once mixed material to depths with $\mathrm{T}>2 \times 10^{6} \mathrm{~K}$. They have presented evidence for ${ }^{6} \mathrm{Li}$ in the star HD 82943 (although the result is disputed; see Reddy et al. (2002)), arguing that it must have been delivered to the stellar surface at some 
point by infalling material. Further support for pollution has been provided by differential Fe and Li abundance enhancements in binary systems (Gratton et al. 2001; Laws \& Gonzalez 2001; Zucker et al. 2002, and references therein), although some of these results have since been reconsidered (Desidera et al. 2004). Binaries may provide an ideal environment for identifying the signature of pollution, since the surface composition of a pollution candidate may be easily compared to that of its companion, which has presumably preserved the system's primordial abundances. Nevertheless, these scenarios still cannot discriminate between accretion of inward-migrating planets and other processes involving much smaller mixes of dust, comets, or planetesimals as the source of metal-rich material.

\subsection{Theoretical Motivations}

To make progress in determining whether abundance enhancements in stars with planets and some binary system members is due to pollution by planetary material, further observations and more constraining theories are required. We propose that such constraints might be provided by detailed modeling of the interiors of stars thought to have accreted high-Z material. We suggest that in depositing heavy metal material in the interiors of stars, pollution should influence stellar structure and evolution in a predictable way that is distinguishable from the behavior of uniform-metallicity stars. Siess \& Livio (1999a,1999b) have developed detailed models of the ingestion of massive planets by red giant and asymptotic giant branch stars. Although some other groups have undertaken hydrodynamical simulations of the stellar interior during consumption of a planet (Sandquist et al. 2002; Hole et al. 2001), the residual effects of small to moderate amounts of metal enrichment on solarlike stars have not been considered in detail. To understand the ramifications of pollution, there is a real need for a precise description of the physical properties involved in planet (or otherwise high-Z material) consumption.

The most quantitative analyses of pollution-induced metallicity enhancement have focused on specific components of stars (e.g., the convection zone), rather than a comprehensive treatment of the physics governing the stellar interior. Such approaches are not self-consistent and may overlook potentially important phenomena that arise in polluted stars. There is no guarantee, for instance, that a metal-enriched convective zone will not alter the entire stellar temperature structure or lead to runaway, nonequilibrium processes. The study of pollution's effects on the stellar interior does have some history when it comes to our Sun. It was suggested by Joss (1974) that substantial contamination of the Sun by infalling debris had masked the composition of the radiative solar interior, following on earlier ideas by Hoyle (1939) and Lyttleton (1936). Dirty solar models were developed by Christensen- 
Dalsgaard et al. (1979) to study the effects on the solar neutrino problem. These models treat the metal-enriched convection zone (both its lower and upper boundaries) in a very simple manner. Recent dirty solar models by Winnick et al. (2002) use the same approach, which brings the star to a new thermal equilibrium by adjusting a free parameter, like the mixing length, that is not constrained by observations. When applied to stars other than the sun, the simple approach of enhancing the metallicity of the surface layers does not allow the star to adjust to a new thermal equilibrium, as Ford, Rasio \& Sills (1999) pointed out. Dotter \& Chaboyer (2003) have recently presented similar polluted stellar evolution models that do not describe the physical changes in the interior structure.

Fully self-consistent polluted stellar evolution models for solar-type stars have not been discussed extensively in the literature. To this end, we have modified an existing accurate stellar evolution code to allow for discontinuities in metallicity inside stars. We aim to examine the effects of differential metal enrichment on stellar structure and evolution, when applied at times close to and after zero-age main sequence (ZAMS). By incorporating a pollution scheme directly into the evolution program, we allow stars that accrete heavy material to undergo a thermal relaxation process prescribed solely by the physics already built into the code, thereby producing one continuous evolutionary model. Our motivation for carrying out these simulations is in the possibility that many stars might have consumed planets during different stages of their evolution; mostly before settling on the ZAMS, but not restricted to that. Since current evidence is limited to stars similar to the sun, we will consider only stellar models in the $0.8-1.4 \mathrm{M}_{\odot}$ range. Such stars have surface convection zones and mixing layers, which play a crucial role in the changes we study. In subsequent sections, we discuss the main features of the resulting polluted stellar evolution models and provide a comprehensive set of evolutionary tracks.

\section{Adding "Metals" to a Surface Convection Zone}

Prior to detailed numerical modeling, we highlight the dominant physical changes expected subsequent to metal enhancement events in convection zones of stars. As discussed in $§ 3.2$, the planetary material is assumed to be confined within the outer convective region of the accreting star. This leads to a discontinuity in relative metal content (by mass), Z, and hence molecular weight, at the convection zone boundary. Assuming Kramer's type bound-free opacity sources typical of lower main sequence stars with $\mathrm{Z} \gtrsim 10^{-4}$, we have $\kappa \propto \mathrm{Z}(1+\mathrm{X}) \rho \mathrm{T}^{-3.5}$, where $\kappa$ is the opacity, and $\mathrm{X}$ is the relative hydrogen content by mass. After enrichment in a convection zone, in which the metal fraction is raised from some value $\mathrm{Z}$ to $\mathrm{Z}+\Delta \mathrm{Z}$, $\mathrm{X}$ will decrease to $\mathrm{X}\left(\frac{1-Z-\Delta Z}{1-Z}\right)$. As long as $\mathrm{Z}$ is not unreasonably large, $\kappa$ will 
then increase, based on its $\mathrm{Z}(1+\mathrm{X})$ dependence. Thus, there should also be a change in opacity at the convection zone boundary. Updated data from the OPAL opacities (Iglesias \& Rogers 1996) suggests that this change should be particularly pronounced near the base of the stellar convection zone, since the opacity is quite sensitive to the Fe abundance at the $\sim 10^{6} \mathrm{~K}$ characteristic temperatures here. The requirements for stability at this location will presumably be affected by the discontinuities in molecular weight and opacity, since the radiative temperature gradient, $\nabla_{\text {rad }}$ is directly proportional to $\kappa$. As established by the Schwarzschild criterion, the onset of convection occurs where the radiative temperature gradient exceeds the adiabatic temperature gradient, $\nabla_{\mathrm{ad}}$. The increase in $\nabla_{\mathrm{rad}}$ at the bottom of the convection region should cause convective instability to extend to lower depths within the star. The related phenomenon of a more shallow convection zone in the absence of high metal content is indeed observed in stars with overall low metallicity (Spite \& Spite 1982).

Pollution-induced changes should not only be isolated to the lower portion of the convection zone. Qualitatively, elevated opacities will restrict the amount of radiation passing from the inner to the outer regions of the star. In general, stars of overall high metallicity occupy main sequence positions on the H-R diagram that are lower in both luminosity and temperature than their low-metallicity counterparts. We expect roughly similar behavior for polluted stars, as they undergo initial adjustments after addition of high-Z material. But since the mass of accreted metals and size of the convection zones are small compared to the overall dimensions of the stars under consideration, pollution may not have any long term effects on the central nuclear burning, and thus the luminosity.

The persistence of elevated opacity in the convection zone should, however, indefinitely maintain a lower effective temperature (due to lower flux) than that of the corresponding unpolluted star. For negligible changes in luminosity, this effect causes an increase in the stellar radius. The expansion can be understood as an adjustment necessary to restore radiative equilibrium in the outer regions of a polluted star by increasing the area over which energy may escape outward.

In summary, the general physical outcomes to be expected from metal pollution include both shifts in lower convection zone limit and changes at the upper boundary, due to enhanced opacity. Such enriched convection zones are hydrostatically stable, and the adjustment of stellar structure is a gradual quasi-equilibrium process (D. Sasselov \& B. Hansen 2003, private communication). 


\section{Polluted Stellar Models: The Modified Computation}

\subsection{The Baseline Stellar Evolution Code}

The baseline code is the same as that used in our recent work (Cody \& Sasselov 2002, hereafter CS02), and based on the Princeton Stellar Evolution Code (Sienkiewicz, Paczynski \& Ratcliff 1988). The program solves the equations of stellar structure on a one-dimensional stellar mass grid, making use of the OPAL radiative opacities (Iglesias \& Rogers 1996; Rogers \& Iglesias 1995), Livermore Laboratory equation of state data (Rogers, Swenson \& Iglesias 1996), and nuclear reaction rates from Bahcall, Pinsonneault, \& Wasserburg (1995). Molecular and grain opacities supplied by Alexander \& Ferguson (1994) are also employed in lower temperature regions of the opacity tables, and equations of state correspond to an ideal or partly ionized gas. In addition to tracking temperature, luminosity, and density evolution, the code traces abundances of $\mathrm{H}, \mathrm{He} 3, \mathrm{He} 4, \mathrm{~N} 14, \mathrm{O} 16$ and $\mathrm{O} 17$ as produced by hydrogen burning in the pp chain and $\mathrm{CNO}$ cycle; in this work, we consider all elements heavier than helium as part of the stellar "metal" content. Diffusion of helium and other elements has not been incorporated into the calculations. Other conditions modeled in the code include slow rigid-body rotation, as well as a simple overshooting scheme. The number of mass points

in the grid is adjustable, and we find that it must be maintained at a minimum of 4000 to provide satisfactory resolution. Limitations in the equation of state tables also restrict models to $\gtrsim 0.87 \mathrm{M}_{\odot}$. We are able to verify the general accuracy of the code in the "allowed" parameter space by comparing an evolutionary track at solar mass and metallicity with the sun's known temperature and luminosity. Good agreement is obtained, and we attribute the slight difference $(\sim 0.2 \%)$ between theoretical and observed parameters to neglect of helium diffusion. We have also run comparisons with the Yale code (see CS02).

\subsection{Assumptions of Star/Planet Interaction}

To trace the effects of heavy-element accretion due to consumption of planets, we have modified the baseline stellar evolution code to allow for non-uniform metallicity distributions within stars. Our models proceed on several assumptions. First, we require that material from inward-migrating planets enters the stellar envelope and dissolves completely within the convection zone, contributing negligible contamination to the inner regions of the star. While there remain many questions about the dynamics of orbital decay both before and after entrance into a host star, recent calculations indicate that complete disintegration of a planet within the convection zone is realistic for a range of planet masses, compositions and orbital time scales. Sandquist et al. $(1998,2002)$ have evaluated the feasibility of this scenario with 
hydrodynamical simulations of giant planet accretion. Testing planet consumption in stars in the neighborhood of $1 \mathrm{M}_{\odot}$, they find that pollution is capable of producing observable surface metallicity enhancements, but the percentage of planetary mass dissolved within the convection zone is highly dependent on the structure of both star and planet. In addition to gas giants, smaller rocky planets and planetesimals have also been cited as potential sources of pollutant material (Gratton et al. 2001, and references therein). Because of the current lack of constraint on amount and type of debris that could be accreted onto stars, pollution is best characterized as a change in metallicity; our models make no prior assumptions about the nature of the polluting planets, except to specify the mass of heavy element enrichment. Furthermore, we restrict the physics to scenarios in which the accreted metal content is mixed evenly throughout the convective region. We note that the extent of observable surface metallicity enhancement is governed by the structure (i.e., depth, density) of the stellar convection zone.

An additional consideration relevant to the evolution of polluted stars is the time of planet accretion. Since the mechanisms of orbital migration are not well understood, no particular time appears most favorable for planetary migration and consumption. A rough lower bound is offered by evidence that any observable pollution takes place in stars that have already reached the main sequence, because the large convection zones of pre-mainsequence stars would lead to considerable dilution of the polluting material (Laughlin 2000; Gonzalez et al. 2001). Consequently, the models produced for this analysis disregard stars that reside on the Hayashi pre-main sequence tracks. In substantially evolved stars, on the other hand, there is no reason to discount the possibility of metal enrichment. Hence, the time scale pertinent to pollution runs from the zero-age main sequence to the subgiant stage. By specifying the stellar age at time of enrichment along with the amount of metallicity enhancement, we are set to describe stellar pollution as a simple two-parameter process.

\subsection{Implementation of the Pollution Scenario}

Under the above considerations, our adaptation of the Princeton Stellar Evolution Code according to pollution theory leaves most of the original physics unaltered. All models employ mixing length theory to compute the convective envelope, incorporating the solar mixing length value of 1.69 (Guenther \& Demarque 1997). In addition, helium content $(\mathrm{Y})$ is varied in accordance with a suitable linear enrichment law, with $\delta \mathrm{Y} / \delta \mathrm{Z}=2.5$, and we have chosen as a zero point the well-known solar values, $\left(\mathrm{Y}_{\odot}, \mathrm{Z}_{\odot}\right)=(0.2741,0.0200)$ (Guenther \& Demarque 1997). Hydrogen, X, is dependent on metallicity and given by the usual equation $X+Y+Z=1$. Primary modifications involved changing the program 
to permit variation of metallicity with stellar radius. We parameterize pollution by $\Delta \mathrm{Z}$, the change in convection zone metal content due to planet consumption, and $t$, time of enrichment (Gyr). Pollution is carried out by uniformly increasing the value of the relative metal abundance $(\mathrm{Z})$ within the confines of the convection zone at the specified time. The code determines these boundaries and the corresponding convection zone mass, $\mathrm{M}_{\mathrm{cz}}$, through the Schwarzschild stability criterion (i.e., radiative temperature gradient equals adiabatic temperature gradient). Although uniform enhancement of convection zone metallicity may not be accurate insofar as mixing requires substantial time, the relevant time scales for planet consumption, as well as radial mixing and meridional circulation are short enough compared to the overall evolutionary time steps that they may be neglected. The former is approximately $\lesssim 10^{7} \mathrm{sec}$ (as found with our code), and the latter is up to $\sim 10^{3}$ yr for the most slowly rotation stars (see Brun \& Toomre 2002, for a discussion of meridional circulation in the sun).

During and after planet accretion, several conditions influence $\Delta \mathrm{Z}$, the value of metal enhancement within the convection zone, as well as the subsequent overall metallicity distribution. Convection zone depth is one crucial factor, and its thickness as a function of total stellar mass is well known. In stars above $1.3 \mathrm{M}_{\odot}$, this region is nearly non-existent, while for stars below $1 \mathrm{M}_{\odot}$, it deepens rapidly. Yet for masses in the range $1.2-1.5 \mathrm{M}_{\odot}$, where the convection zone is predicted to be very thin, further mixing is observed, as in the "Lithium dip" present in Hyades stars (Boesgaard \& Tripicco 1986). This phenomenon has largely been attributed to stellar rotation (Boesgaard \& King 2002). But since it is not well accounted for in standard stellar evolution theory, we only employ the code in modeling pollution for stars below $1.2 \mathrm{M}_{\odot}$. In this range, convection zone depth monotonically decreases with increasing mass. As Pinsonneault, DePoy \& Coffee (2001) and others point out, pollution should be most easily observable in stars at the high-mass end, because there will be little dilution of metal enrichment within their thin convection zones. Since we have chosen to give $\Delta \mathrm{Z}$ a priori for each model, without regard to size of the convection zone, this simply means that the corresponding amount of pollutant material needed will be smaller in models of higher overall mass. For a given $\Delta \mathrm{Z}$, conversion from metal enrichment value to pollutant mass is carried out by taking into account the mass enclosed within the convection zone limits, $\mathrm{M}_{\mathrm{cz}}$. Using the code, we derive this quantity as a function of overall stellar mass, as shown at age $\log (\mathrm{t})=5$ (due to the code time step, we cannot produce the zero-age convection zone) in Fig. 1. Ignoring additional mixing evident in the "lithium dip," this is consistent with the results of Murray et al. (2001).

Of lesser importance in determining the metallicity distribution are progressive shifts in the convective boundary. Although the initial amount of metal throughout the entire star $(Z)$ and within the convection zone $(Z+\Delta Z)$ is fixed in each model, expansion of this 
region leads to slight dilution of the enriched material. Slow changes in the boundary are typical during stellar evolution but, as discussed in $\$ 2.2$, they should be more pronounced after addition of high-metallicity material, as a result of increased opacity. The modified version of the code has been designed to recalculate the metallicity-dependent opacities and equations of state at each location in the stellar grid where $\mathrm{Z}$ differs significantly from that of neighboring points. Interpolation is performed between $(X, Z)$ entries in the equation of state and opacity tables to provide the most accurate values. When pollution induces a downward shift of the convection zone boundary, the metal-enhanced region is mixed with the layer of elements below. As during the initial pollution event, re-mixing of the convection zone contents takes place during each single time-step of the simulation. This deepening of the convection zone is followed by the code and tends to reduce the observed metallicity enrichment due to pollution.

Overshooting is an additional effect that counteracts the relative metal enrichment generated by pollution in the upper layers of the star. Within the evolution code, the top and bottom of the convection zone are determined with the Schwarzschild criterion; in reality, we expect substantial quantities of higher metallicity material to be transported beyond the convective boundaries. A simple overshooting model was implemented in the baseline code to account for this phenomenon. During the majority of our pollution simulations, enriched material is not only circulated within the convection zone, but is also efficiently mixed throughout the atmosphere above. Combined with the consequences of a deepening of the convection zone, or an initially large convective depth, overshooting has the potential to reduce $\Delta \mathrm{Z}$ from its initial value. In Fig. 2 , we show radial metallicity profiles for a $1 \mathrm{M}_{\odot}$ at several different times, both before and after pollution; gradual dilution due to these effects is evident. Ignoring any mass loss or mixing in semi-convective regions, no other processes contribute to significant dilution of the higher metallicity region. We confirm the accuracy of the modified code in the limiting case $\Delta \mathrm{Z}=0$ by showing that it produces the same evolution tracks as the original program. The results of polluted evolution, however, are much more difficult to verify, since there are no precedents for this sort of stellar model in the literature.

\section{Pollution: General Trends}

\subsection{Convection Zone Changes}

Before analyzing full stellar models, we investigated differences in the convection zone boundaries of stars with and without pollution. As previously discussed, we expect a deepening of the lower convection zone boundary, due to increased opacity. We have modeled

both the mass contained within polluted and unpolluted convection zones, as well as the 
radii of their boundaries. As seen in Fig. 3, the top of the convection zone extends outward nearly to the stellar surface. Upon pollution with planetary material, a star responds by increasing its radius, whereas the inner boundary of the convection zone remains at virtually the same absolute distance from the core. The combination of these two effects leads to a relative deepening of the convection zone.

The behavior of the convection zone mass is also in line with our theoretical predictions. Depicted in Fig. 4, it displays a marked increase in stars of significant convective depth (i.e., those with $1.0 \mathrm{M}_{\odot}, 0.9 \mathrm{M}_{\odot}$, and lower). The enclosed amount of mass is linked with the radial changes, but tied to the stellar density profile as well. Thus, in the $1.1 \mathrm{M}_{\odot}$ model, the polluted convection zone is actually a bit smaller than its unpolluted counterpart; this is the outcome of the larger stellar radius and correspondingly decreased density near the surface. In addition, there is an obvious expansion of the convection zone as model stars approach the subgiant branch; this can be seen if both Fig. 4 and Fig. 3.

\subsection{Polluted Stellar Evolution Tracks}

We have calculated polluted stellar evolution tracks for a variety of stars from $\mathrm{M}=0.9$ $1.2 \mathrm{M}_{\odot}$ and $\mathrm{Z}=0.01-0.03$. For models below $0.85 \mathrm{M}_{\odot}$, the convection zone becomes quite deep, and effects of pollution are not expected to be significant. For purposes of comparison, most tracks were calculated assuming pollution at the zero-age main sequence. However, an additional set of models was produced to explore the outcome of pollution at much later times, from $2 \times 10^{5}$ yr to 9 Gyr. We now discuss several notable trends present among the polluted stellar evolution tracks.

As seen if Figs. 5 and Fig. 6, pollution causes evolution tracks to shift downward in temperature. This shift is the main feature of the thermal adjustment process resulting from the addition of heavy-metal material to the outer zones of a star. The duration of adjustment is given roughly by the thermal relaxation timescale (see Stehle \& Ritter 1999, for a detailed discussion of this phenomenon as expected in accretion of material onto the secondary star of a cataclysmic variable system), so the full extent of the temperature shift is completed over only one timestep within the evolution models. Our results are consistent with the models of Dotter \& Chaboyer, which require that additional metals be introduced over several timesteps; they find that polluted stars are hotter than unpolluted stars with the same mass, age, and metallicity.

For all test cases from $\mathrm{M}=0.9-1.2 \mathrm{M}_{\odot}$ and $\mathrm{Z}=0.01-0.03$, the temperature shift is roughly proportional to the parameter $\Delta \mathrm{Z}$. While the proportionality factor varies with mass, this 
can be attributed to the fact that size of the convection zone is directly related to stellar mass. For constant values of $\Delta \mathrm{Z}$, the physical amount of metals added to the convection zone will depend on the mass of the convection zone. Thus, for higher masses, the convection zone is smaller, less pollutant material is introduced, and hence the effect is smaller. Aside from this trend, the only factor determining the magnitude of temperature shifts from model to model seems to be the value of $\Delta \mathrm{Z}$. The remarkable consistency in this result could be useful in discriminating between pollution or lack thereof, if the polluted version of the code is applied to stars known to harbor planets.

Another feature verified by the computer modeling is that pollution-induced shifts in evolutionary tracks are independent of when pollution happens, for times after the late as the ZAMS age and before the subgiant stage. Polluted models were calculated for a $1.1 \mathrm{M}_{\odot}$ star starting at solar metallicity, and polluted by $\Delta \mathrm{Z}=0.01$. When pollution was delayed until the arbitrary times of $2.1 \times 10^{5}, 1.0 \times 10^{6}, 1.0 \times 10^{8}, 9.0 \times 10^{8}$, and $6.25 \times 10^{9} \mathrm{yr}$, the polluted and unpolluted portions of each track remained coincident with each other (see Fig. 7). We conclude that a polluted star will indeed reach the same equilibrium state, regardless of whether high-metallicity material is acquired immediately, or at a later time when the star has evolved a bit. This result indicates that pollution of the surface convection zone has ltitle to no effect on the central burning process, and reinforces the simplicity of modeling polluted stars, since we are now able to omit the time of pollution as a free parameter.

\subsection{Application to HD 209458}

As a test of the possible applications to our modified stellar evolution code, we consider

the case of the star HD 209458, host to a transiting planet (see CS02). At $[\mathrm{Fe} / \mathrm{H}]=0.0$, the star does not appear to be particularly enriched with metals. Nevertheless, if we assume that the majority of stars with planets fall on an enhanced metallicity distribution a full 0.2 dex higher than "ordinary" solar neighborhood stars, then it is not unreasonable to suppose that HD 209458 was polluted from an original value of $[\mathrm{Fe} / \mathrm{H}]=-0.2$. There is further uncertainty, however, in the conversion from initial $[\mathrm{Fe} / \mathrm{H}]$ to initial $\mathrm{Z}$, since the iron content of pollutant is not known. We explore the range of possibilities by considering two cases: 100\% iron pollution, and a mix of metals in the same proportions as found in the sun (in which iron comprises $7.18 \%$ of metals by mass; Grevesse \& Noels (1993)). These correspond to initial Z values of 0.019 and 0.013 , respectively. The amounts of metal pollution $\Delta \mathrm{Z}$ needed to raise the initial $\mathrm{Z}$ to 0.02 on the surface are then 0.001 and 0.008 (allowing for some dilution of the metals due to overshooting and expansion of the convection zone).

For the unpolluted case $(\mathrm{Z}=0.02, \Delta \mathrm{Z}=0.0)$ as well as the two polluted cases, we have 
computed evolution tracks to match HD 209458's observed temperature of $\log \left(\mathrm{T}_{\text {eff }}\right)=3.778$ and luminosity $\log \left(\mathrm{L} / \mathrm{L}_{\odot}\right)=0.208$. The best-fit models are illustrated in Fig. 8. Further models were fit to the edges of the $\mathrm{T}_{\text {eff }} \mathrm{L}$ error box to determine the range of possible masses and ages for the two scenarios, as given in Table 1. When metallicity is also varied according to $[\mathrm{Fe} / \mathrm{H}]=0.0 \pm 0.2$ (or $\mathrm{Z}=0.02 \pm 0.005$ ), the mass uncertainty increases to approximately \pm 0.10 .

\begin{tabular}{ccccc}
\hline \hline Model & $\begin{array}{c}\text { Mass } \\
\left(\mathrm{M} / \mathrm{M}_{\odot}\right)\end{array}$ & $\begin{array}{c}\text { Age } \\
(\mathrm{Gyr})\end{array}$ & $\begin{array}{c}\text { Initial } \\
{[\mathrm{Fe} / \mathrm{H}]}\end{array}$ & $\begin{array}{c}\text { Initial Convective } \\
\text { Mass }\left(\mathrm{M} / \mathrm{M}_{\odot}\right)\end{array}$ \\
\hline Unpolluted & $1.06_{-0.02}^{+0.03}$ & $5.7_{-2.5}^{+1.5}$ & 0.0 & $1.8 \times 10^{-2}$ \\
Polluted: Metals in & $0.97 \pm 0.02$ & $6.6_{-2.0}^{+1.5}$ & -0.2 & $1.5 \times 10^{-2}$ \\
Solar Proportions & & & & $1.8 \times 10^{-2}$ \\
Polluted: $100 \% \mathrm{Fe}$ & $1.06_{-0.02}^{+0.03}$ & $5.6_{-2.5}^{+1.5}$ & -0.2 & \\
\hline \hline
\end{tabular}

Table 1: Best-fit parameters for HD 209458 based on $\log \left(\mathrm{T}_{\text {eff }}\right)=3.778 \pm 0.004$, $\log \left(\mathrm{L} / \mathrm{L}_{\odot}\right)=0.208 \pm 0.040$, and $\mathrm{Z}_{\text {surface }}=0.02 \pm 0.005($ see CS02)

Assuming the solar mixing length, 1.69 (Guenther \& Demarque 1997) and helium $(\mathrm{X}=0.70)$, we find a reduction in mass by $0.09 \mathrm{M}_{\odot}$ and increase in age by 1.4 Gyr from the best-fit unpolluted values. For the polluted model with solar metal composition, we find $\mathrm{M}=0.97 \mathrm{M}_{\odot}$ and age $6.6 \mathrm{Gyr}$. This value is at the very edge of allowable masses found by CS02, taking into account the full range of uncertainties in HD 209458's temperature, luminosity, and metallicity in the unpolluted scenario. Given the size of the star's convection zone, pollution by $\sim 40 \mathrm{M}_{\oplus}$ of metal material is required to produce the presumed metallicity enhancement of $\Delta \mathrm{Z}=0.008$. If this were the case, orbital inclination estimates for the planet HD 209458b would need to be revised to match the lower mass of its parent star. Following the $\chi^{2}$ lightcurve fit procedures described in CS02, the resulting most probable planetary radius would be unaltered, but the mass uncertainty would actually decrease by $\sim 5 \%$. However, the fit of the predicted lightcurve produced by a $1.42 \mathrm{M}_{\mathrm{J}}$ planet to the observed one is not as good for a $0.97 \mathrm{M}_{\odot}$ parent star (the reduced $\chi^{2}$ value increases from 2.5 to 2.9$)$.

For $100 \%$ iron pollution, on the other hand, the best-fit polluted and unpolluted stellar evolution tracks are virtually indistinguishable. This is because only $\sim 4 \mathrm{M}_{\oplus}$ of pure iron 
pollution is required to raise the metallicity from $[\mathrm{Fe} / \mathrm{H}]=-0.2$ to $[\mathrm{Fe} / \mathrm{H}]=0.0$. The resulting $\Delta \mathrm{Z}$ of 0.001 is not large enough to substantially alter the stellar parameters.

\section{Discussion}

Our numerical calculations have confirmed the theoretical expectation that pollution is capable of causing significant changes in the evolution of stars. We have shown that pollution from a primordial metal fraction $Z_{\circ}$ to a final value $Z_{\circ}+\Delta Z$ shifts evolution toward lower temperatures, but the resulting evolutionary tracks are not coincident with models of uniform metal fraction $\mathrm{Z}_{\circ}+\Delta \mathrm{Z}$. In fact, the polluted tracks lie much closer on the H-R diagram to the $\mathrm{Z}=\mathrm{Z}_{\circ}$ models than those with $\mathrm{Z}=\mathrm{Z}_{\circ}+\Delta \mathrm{Z}$ throughout the entire star. In Fig. 9, we illustrate this effect for three stars of mass $1.0 \mathrm{M}_{\odot}$. The upshot of this result is that if a star of a particular mass is suspected of having accreted a planet, its position on the H-R diagram should not be consistent with the observed metallicity. However, because accurate stellar masses are rarely available, this condition may not be such a good discriminant for the pollution scenario. Nevertheless, it could be useful in cases where additional constraints are available, such as stellar clusters, where isochrones fix the mass for a given temperature and luminosity.

Having explored the correlation between $\mathrm{M}_{\mathrm{cz}}$, amount of metal enhancement, and stellar temperature, we can address the prospects of directly observing abundance changes due to pollution, and the quantities of material required to bring these enhancements about. A number of authors have quoted figures on the order of a few $\mathrm{M}_{\oplus}$ in their estimates of pollution mass (Murray et al. 2001; Murray \& Chaboyer 2002; Pinsonneault et al. 2001). But from the example of HD 209458, it is evident that the amount of mass required is highly dependent on the iron content of the material. If pure metal pollution with iron and other species in solar proportions is added to a convection zone of mass $\mathrm{M}_{\mathrm{cz}}$, the total amount of pollution needed to raise the metal fraction from $\mathrm{Z}$ to $\Delta \mathrm{Z}$ is given by $\mathrm{M}_{\text {poll }}=\mathrm{M}_{\mathrm{cZ}} \Delta \mathrm{Z} /(1-\mathrm{Z}-\Delta \mathrm{Z})$. Thus, for a $1.15 \mathrm{M}_{\odot}$ star with $\mathrm{M}_{\mathrm{cz}} \sim 0.007 \mathrm{M}_{\odot}$ (as shown in Fig. 1), and pollution from $\mathrm{Z}_{\odot}=0.02$ to 0.03 , we find $\mathrm{M}_{\text {poll }} \sim 25 \mathrm{M}_{\oplus}$. And as seen in the hypothetical scenario for HD 209458 $\left(\mathrm{M}_{\text {poll }} \sim 40 \mathrm{M}_{\oplus}\right)$, the necessary $\mathrm{M}_{\text {poll }}$ would be somewhat larger. On the other hand, if the metal pollutant is more iron rich than solar material, as little as one tenth of this mass would be needed to achieve the same surface metallicity enhancements.

The question of what sort of planetary bodies are most likely to serve as contributors to pollution is even more uncertain. Recent theories have proposed that gas giant planets with rocky cores may experience orbital migration and possibly fall into their host stars. But the amount of metal available in the cores of these Jupiter-like objects is not well known, 
even for members of our own solar system. Models developed by (Guillot et al. 1997) and (Guillot 1999) estimate that Jupiter may possess a rocky core of $<12 \mathrm{M}_{\oplus}$, and it contains a total heavy-element mass between 11 and $45 \mathrm{M}_{\oplus}$. Yet, even if a planet with a metallic core of $12 \mathrm{M}_{\oplus}$ were to pollute its host star, the gaseous atmosphere would also supply an amount of hydrogen and helium, thereby diluting the contribution of high-Z material. In the case of Jupiter the average $\mathrm{Z}$ is between $\sim 3$ and 13\%, and for Saturn, it is $\sim 20-30 \%$ (Hubbard et al. 1999). Hence, due to the $\gtrsim 70 \%$ contribution of hydrogen and helium, the mass of accreted planetary matter involved in pollution will inevitably be greater than the values quoted in our pure metal enrichment models. One way around the issue of limited metal content in gas giant planets is ingestion of smaller planetesimals. Because most planets systems are thought to have formed in a circumstellar dust disk, there may be enough rocky material available to pollute stars during the first 10 million years of their lifetimes, before the disk dissipates (Gonzalez 1997). Accretion of planetesimals certainly seems feasible, but the large convection zone depths in very young stars poses problems regarding the observability of such an effect (Murray et al. 2001). Hence, at present, there does not appear to be a universally favored method for large quantities of heavy-element material to accrete onto stars and remain reasonably concentrated at the stellar surface.

Finally, we examine the viability of pollution in light of current observational data on samples of SWPs. Exploring model behavior for a range of masses and fixed $\mathrm{M}_{\text {poll }}$, rather than fixed $\Delta \mathrm{Z}$ (as in Fig. 5) enables us to predict what trends, if any, should be present among the observations if pollution is indeed a common process. We have polluted solarmetallicity $\left(\mathrm{Z}_{\circ}=0.02\right)$ stars on the ZAMS with $10 \mathrm{M}_{\oplus}$ and $40 \mathrm{M}_{\oplus}$, and subsequently evolved them to 3 Gyr. Fig. 10 depicts the temperatures and surface metallicities expected at several different ages. This is similar to Fig. 2 in Pinsonneault et al. (2001), but our models include polluted evolution. Stars with higher temperatures, and hence larger masses, have smaller convection zones; thus is it no surprise that we find significant increases in surface metallicity for the high-temperature models. For comparison, we have overplotted data points from a recent study by Fischer \& Valenti (2003). Pollution would undoubtedly contribute some range of $M_{\text {poll }}$ to stars, as opposed to the fixed quantities $10 \mathrm{M}_{\oplus}$ and $40 \mathrm{M}_{\oplus}$ that we have selected. We have also simplified the situation by only showing stellar models that have initial metal compositions of $\mathrm{Z}_{\circ}=\mathrm{Z}_{\odot}=0.02$. But a superposition of $[\mathrm{Fe} / \mathrm{H}]$ versus $\mathrm{T}_{\text {eff }}$ models covering a range of initial metallicities and pollution masses should still produce preferential surface metallicity enhancement among the high-temperature data points if planet accretion is widespread. No such trend is evident. The presence of older, cooler stars in the data could also skew the results, since the convection zones of evolved stars are deeper, and hence the effects of pollution would be diluted. But the sample here includes only five subgiants, and Fischer \& Valenti (2003) note that none of them have particularly low metallicities. Thus 
the current data suggest that pollution cannot be the only cause behind the relatively high metallicities of stars with planets.

\section{Conclusions}

In modifying the Princeton Stellar Evolution code, we have paved the way for detailed study of stellar structure and evolution as affected by planet accretion. Grids of polluted models for various masses and metallicities have enabled us to identify the main physical outcomes of this process. The most prominent features are:

- Deepening of the stellar convection zone

- Expansion of the star, leading to lower effective temperatures at approximately constant luminosity

- Age-independence of the results

We expect that the general trends presented here will be useful in future attempts to discriminate between pollution and other sources of metal enhancement in stars with planets. In addition, they should be particularly well suited to studies of pollution binary systems or star clusters in which additional parameters constrain stellar age and mass. Pollution models applied to specific candidates in which there is information on the stellar mass or age could also offer fruitful methods of determining whether planet consumption was part of a star's past. In subsequent work, we develop polluted models of several SWPs. We will present predictions for p-mode oscillation frequencies in these systems, with the goal of directly testing for pollution with current asteroseismology missions.

AMC is grateful for support from the Harvard College Research Program throughout this work. We acknowledge additional support from the Harvard Clark Fund. Sincere thanks also go to Douglas Gough and to the referee for useful feedback. 


\section{REFERENCES}

Alexander, D. R. \& Ferguson, J. W. 1994, ApJ, 437, 879

Bahcall, J. N., Pinsonneault, M. H., \& Wasserburg, G. J. 1995, Reviews of Modern Physics, 67,781

Boesgaard, A. M. \& King, J. R. 2002, ApJ, 565, 587

Boesgaard, A. M. \& Tripicco, M. J. 1986, ApJ, 302, L49

Brun, A. S. \& Toomre, J. 2002, ApJ, 570, 865

Castro, S., Rich, R. M., Grenon, M., Barbuy, B., \& McCarthy, J. K. 1997, AJ, 114, 376

Christensen-Dalsgaard, J., Gough, D. O., \& Morgan, J. G. 1979, A\&A, 73, 121

Cody, A. M. \& Sasselov, D. D. 2002, ApJ, 569, 451

Desidera, S., Gratton, R., Scuderi, S., Claudi, R., Cosentino, R., Barbieri, M., Bonanno, G., Carretta, E., Endl, M., Lucatello, S., Fiorenzano, A. F. M., \& Marzari, F. 2004, A\&A, in press (astro-ph/0403051

Dotter, A. \& Chaboyer, B. 2003, ApJ, 596, 496

Fischer, D. A. \& Valenti, J. A. 2003, in ASP Conf. Series 294, Scientific Frontiers in Research on Extrasolar Planets, ed. D. Deming and S. Seager (San Francisco: ASP)

Ford, E. B., Rasio, F. A., \& Sills, A. 1999, ApJ, 514, 411

Gonzalez, G. 1997, MNRAS, 285, 403

-. 1998, A\&A, 334, 221

—. 2003, Reviews of Modern Physics, 75, 101

Gonzalez, G., Laws, C., Tyagi, S., \& Reddy, B. E. 2001, AJ, 121, 432

Gonzalez, G., Wallerstein, G., \& Saar, S. H. 1999, ApJ, 511, L111

Gratton, R. G., Bonanno, G., Claudi, R. U., Cosentino, R., Desidera, S., Lucatello, S., \& Scuderi, S. 2001, A\&A, 377, 123

Grevesse, N. \& Noels, A. 1993, in Origin and Evolution of the Elements, ed. N. Prantzos, E. Vangioni-Flam, \& M. Casse (Cambridge: Cambridge University Press), 14 
Guenther, D. B. \& Demarque, P. 1997, ApJ, 484, 937

Guillot, T. 1999, Science, 286, 72

Guillot, T., Gautier, D., \& Hubbard, W. B. 1997, Icarus, 130, 534

Hahn, J. M. \& Malhotra, R. 1999, AJ, 117, 3041

Hole, K. T., Sasselov, D. D., Kondratko, P. T., Heinke, C. O., Schnee, S., Bonanos, A. Z., \& Ball, G. 2001, BAAS, 33, 4

Hoyle, F. 1939, The Observatory, 62, 217

Hubbard, W. B., Guillot, T., Marley, M. S., Burrows, A., Lunine, J. I., \& Saumon, D. S. 1999, Planet. Space Sci., 47, 1175

Iglesias, C. A. \& Rogers, F. J. 1996, ApJ, 464, 943

Israelian, G., Santos, N. C., Mayor, M., \& Rebolo, R. 2001, Nature, 411, 163

-. 2003, A\&A, 405, 753

Joss, P. C. 1974, ApJ, 191, 771

Laughlin, G. 2000, ApJ, 545, 1064

Laughlin, G. \& Adams, F. C. 1997, ApJ, 491, L51

Laws, C. \& Gonzalez, G. 2001, ApJ, 553, 405

Lin, D. C. 1997, in IAU Colloq. 163, Accretion Phenomena and Related Outflows, ed. D. T. Wickramasinghe, G. V. Bicknell, \& L. Ferrario (ASP Conf. Ser. 121; San Francisco: ASP), 321

Lin, D. N. C., Bodenheimer, P., \& Richardson, D. C. 1996, Nature, 380, 606

Lissauer, J. J. \& Stewart, G. R. 1993, in Protostars and Planets III, ed. E.H. Levy \& J. Lunine (Tucson: University of Arizona Press), 1061

Lyttleton, R. A. 1936, MNRAS, 96, 559

Mayor, M. \& Queloz, D. 1995, Nature, 378, 355

Murray, N. \& Chaboyer, B. 2002, ApJ, 566, 442

Murray, N., Chaboyer, B., Arras, P., Hansen, B., \& Noyes, R. W. 2001, ApJ, 555, 801 
Pinsonneault, M. H., DePoy, D. L., \& Coffee, M. 2001, ApJ, 556, L59

Pollack, J. B., Hubickyj, O., Bodenheimer, P., Lissauer, J. J., Podolak, M., \& Greenzweig, Y. 1996, Icarus, 124, 62

Reddy, B. E., Lambert, D. L., Laws, C., Gonzalez, G., \& Covey, K. 2002, MNRAS, 335, 1005

Rogers, F. J. \& Iglesias, C. A. 1995, in ASP Conf. Ser. 78, Astrophysical Applications of Powerful New Databases, ed. S.J. Adelman \& W.L. Wiese (San Francisco: ASP), 31

Rogers, F. J., Swenson, F. J., \& Iglesias, C. A. 1996, ApJ, 456, 902

Sandquist, E., Taam, R. E., Lin, D. N. C., \& Burkert, A. 1998, ApJ, 506, L65

Sandquist, E. L., Dokter, J. J., Lin, D. N. C., \& Mardling, R. A. 2002, ApJ, 572, 1012

Santos, N. C., Israelian, G., \& Mayor, M. 2001, A\&A, 373, 1019

Sienkiewicz, R., Paczynski, B., \& Ratcliff, S. J. 1988, ApJ, 326, 392

Siess, L. \& Livio, M. 1999a, MNRAS, 304, 925

-. 1999b, MNRAS, 308, 1133

Spite, F. \& Spite, M. 1982, A\&A, 115, 357

Stehle, R. \& Ritter, H. 1999, MNRAS, 309, 245

Trilling, D. E., Benz, W., Guillot, T., Lunine, J. I., Hubbard, W. B., \& Burrows, A. 1998, ApJ, 500, 428

Winnick, R. A., Demarque, P., Basu, S., \& Guenther, D. B. 2002, ApJ, 576, 1075

Zucker, S., Naef, D., Latham, D. W., Mayor, M., Mazeh, T., Beuzit, J. L., Drukier, G., Perrier-Bellet, C., Queloz, D., Sivan, J. P., Torres, G., \& Udry, S. 2002, ApJ, 568, 363 


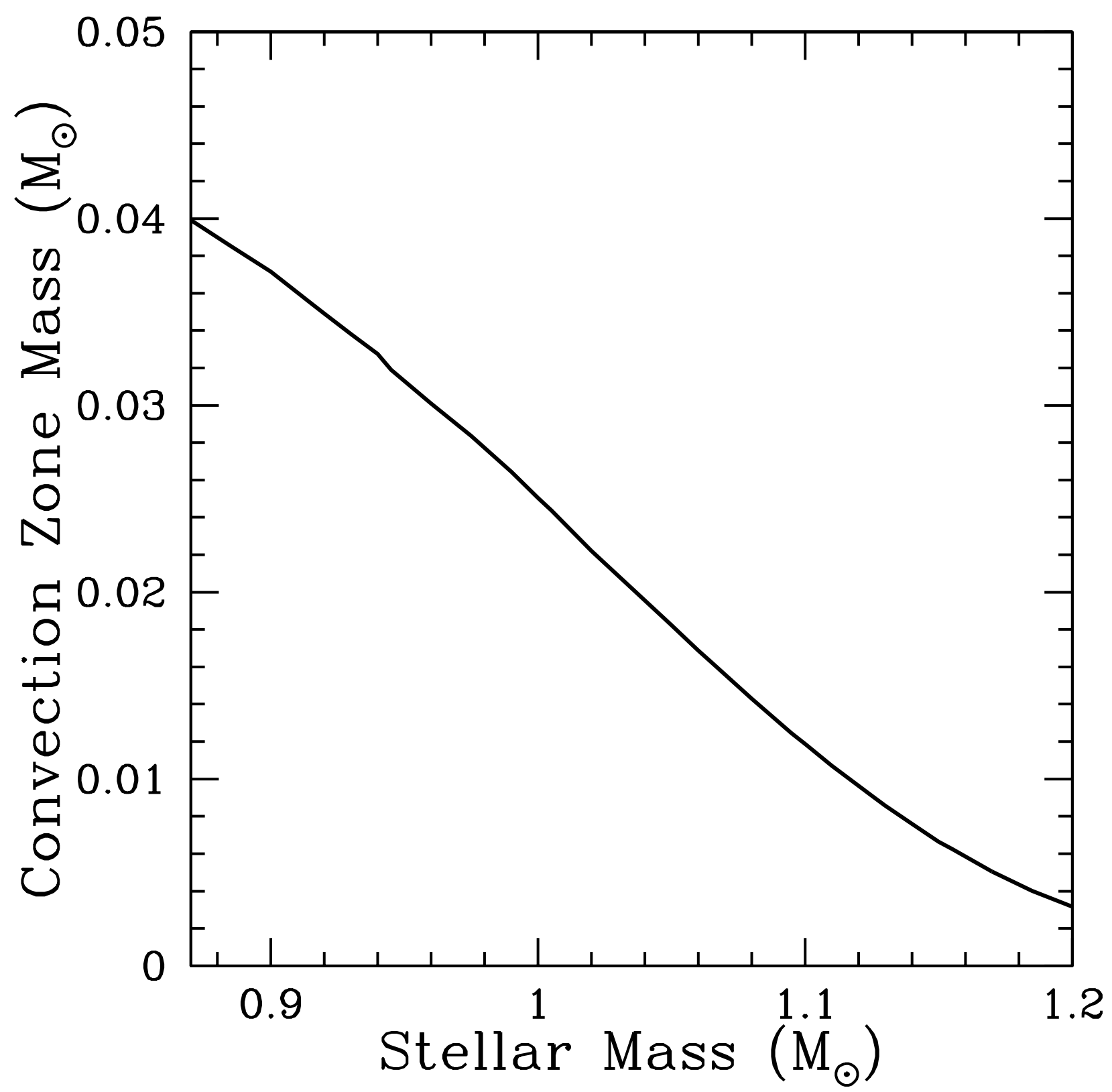

Fig. 1.- Theoretical predictions for stellar convection zone mass as a function of total stellar mass for stars at age $10^{5}$ years. 


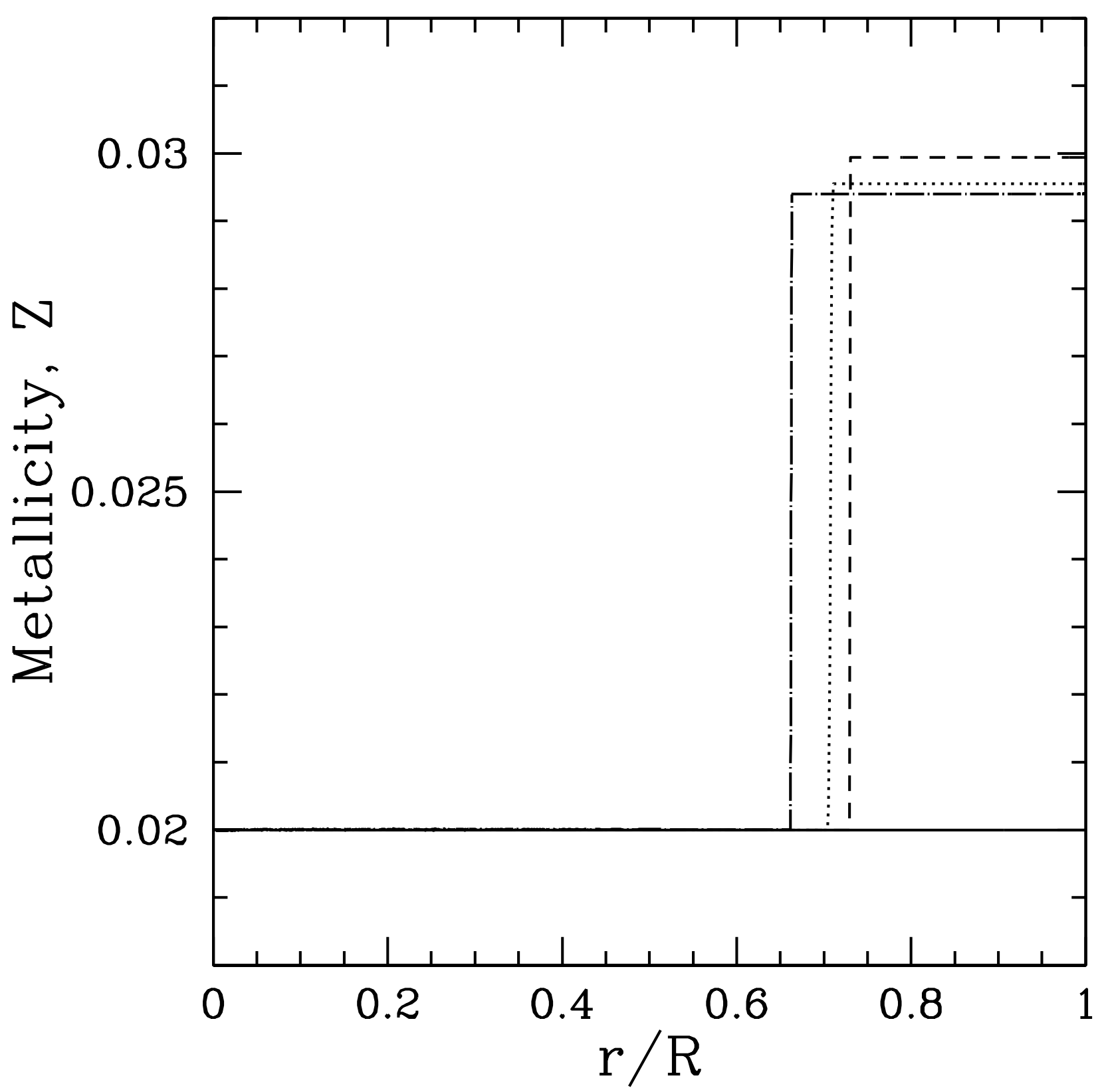

Fig. 2.- For a $1.0 \mathrm{M}_{\odot}$ star polluted from $\mathrm{Z}_{\circ}=0.02$ to $0.03, \mathrm{Z}$ is shown as a function of radius at ages $\mathrm{t}=10^{5}$ (solid line), $10^{5.2}$ (long dashes), $10^{9}$ (dots), and $10^{10} \mathrm{Gyr}$ (dot-dash) 


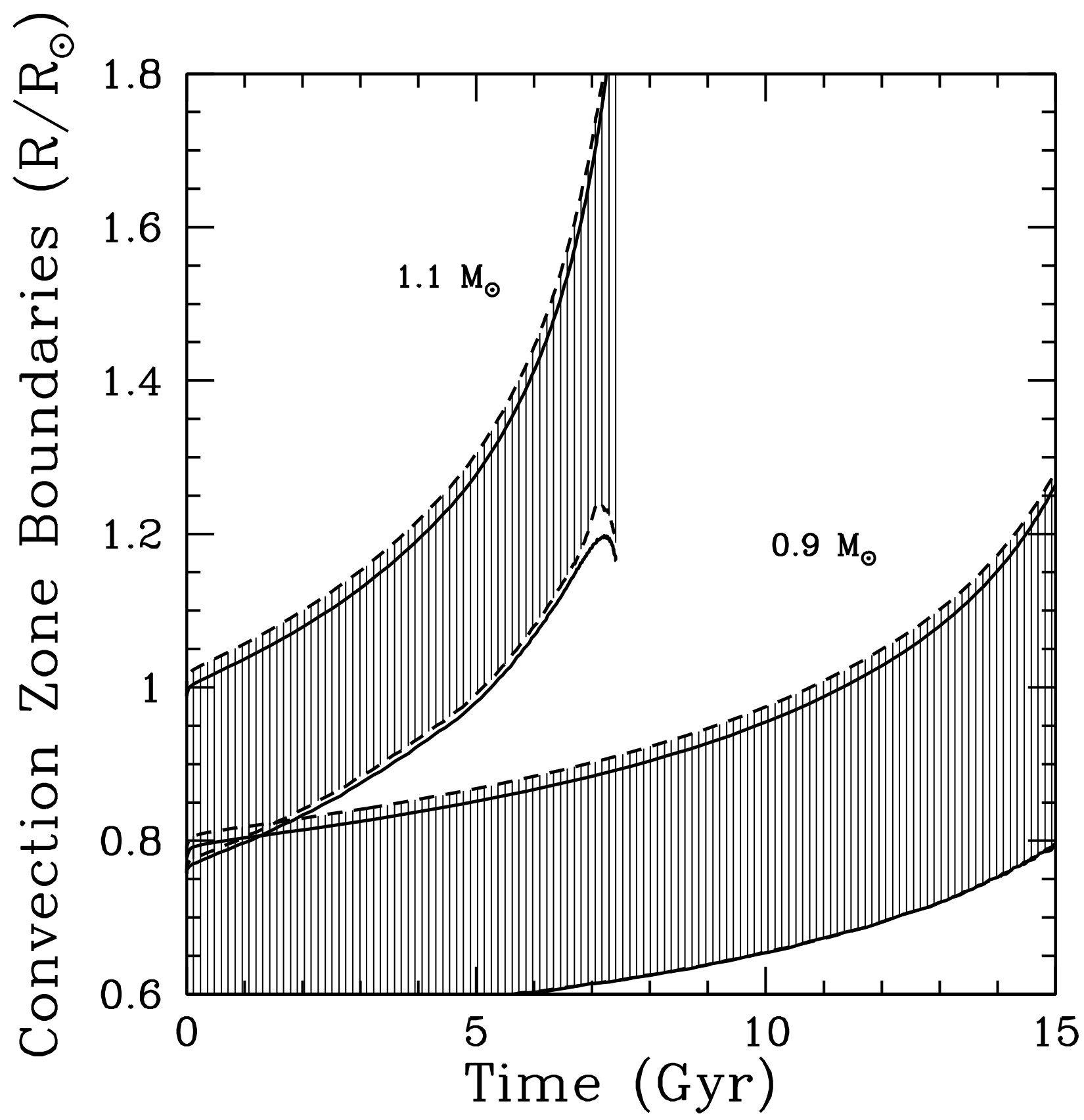

Fig. 3.- Radial boundaries of the stellar convection zone versus time are plotted for polluted and unpolluted models with $\mathrm{X}=0.7, \mathrm{Z}=0.02$, and masses $1.1 \mathrm{M}_{\odot}$ and $0.9 \mathrm{M}_{\odot}$. Convection zone boundaries of polluted models are marked with dashes and were produced with $\Delta \mathrm{Z}=0.01$; boundaries for the unpolluted models are marked as solid lines. Vertical shading shows the extent of each convection zone for clarity; the outer radius of the star is not drawn, because it is within $0.5 \%$ of the convective region upper boundary. 


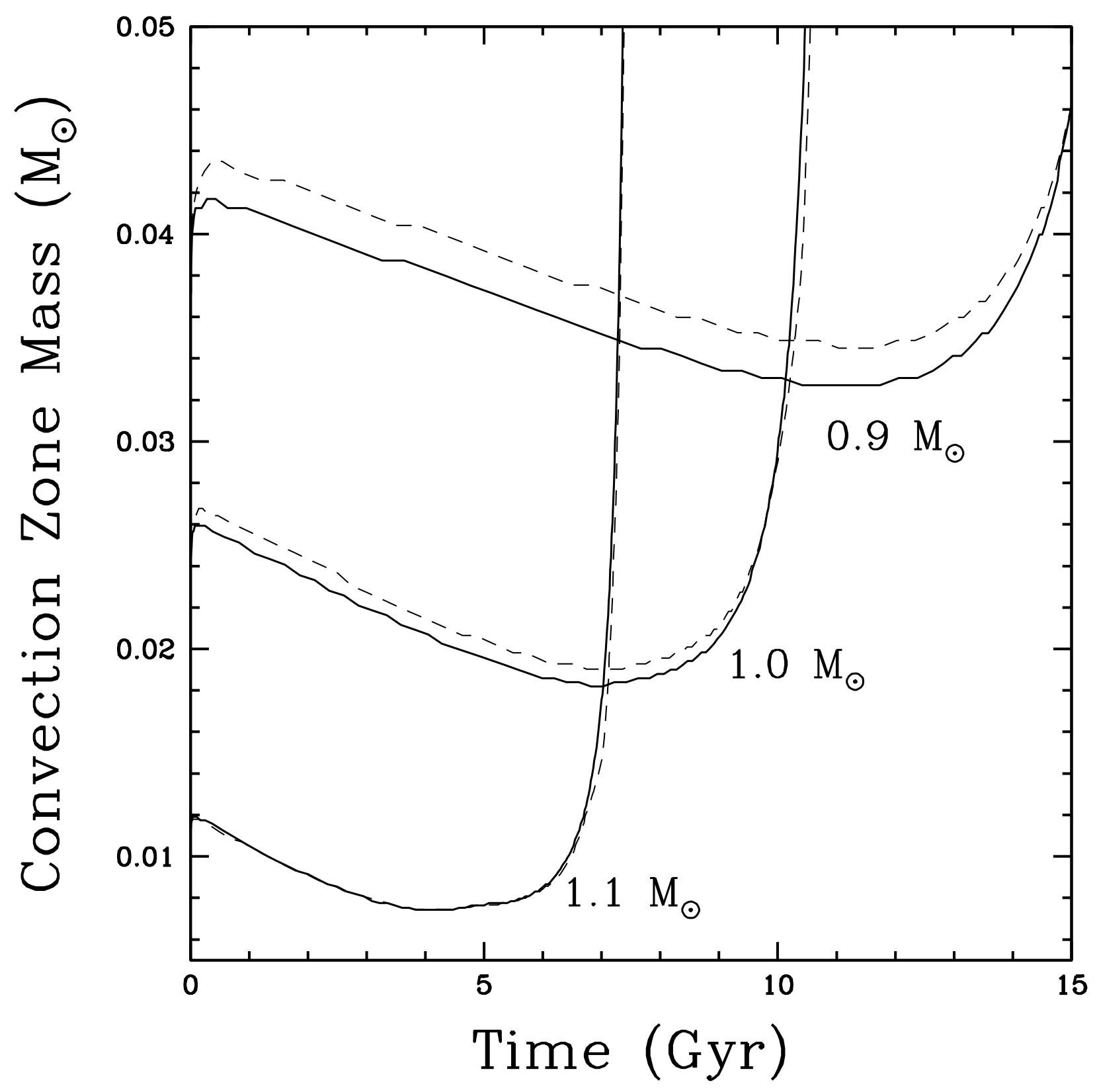

Fig. 4.- Mass of the stellar convection zone versus time is plotted for polluted and unpolluted models with $\mathrm{Z}=0.7, \mathrm{Z}=0.02$, and masses $1.1 \mathrm{M}_{\odot}, 1.0 \mathrm{M}_{\odot}, 0.9 \mathrm{M}_{\odot}$. Polluted models are marked with dashes and were produced with the specification $\Delta \mathrm{Z}=0.01$. Small ridges on the curves are due to limited mass resolution and are not true features of the convection zone evolution. 


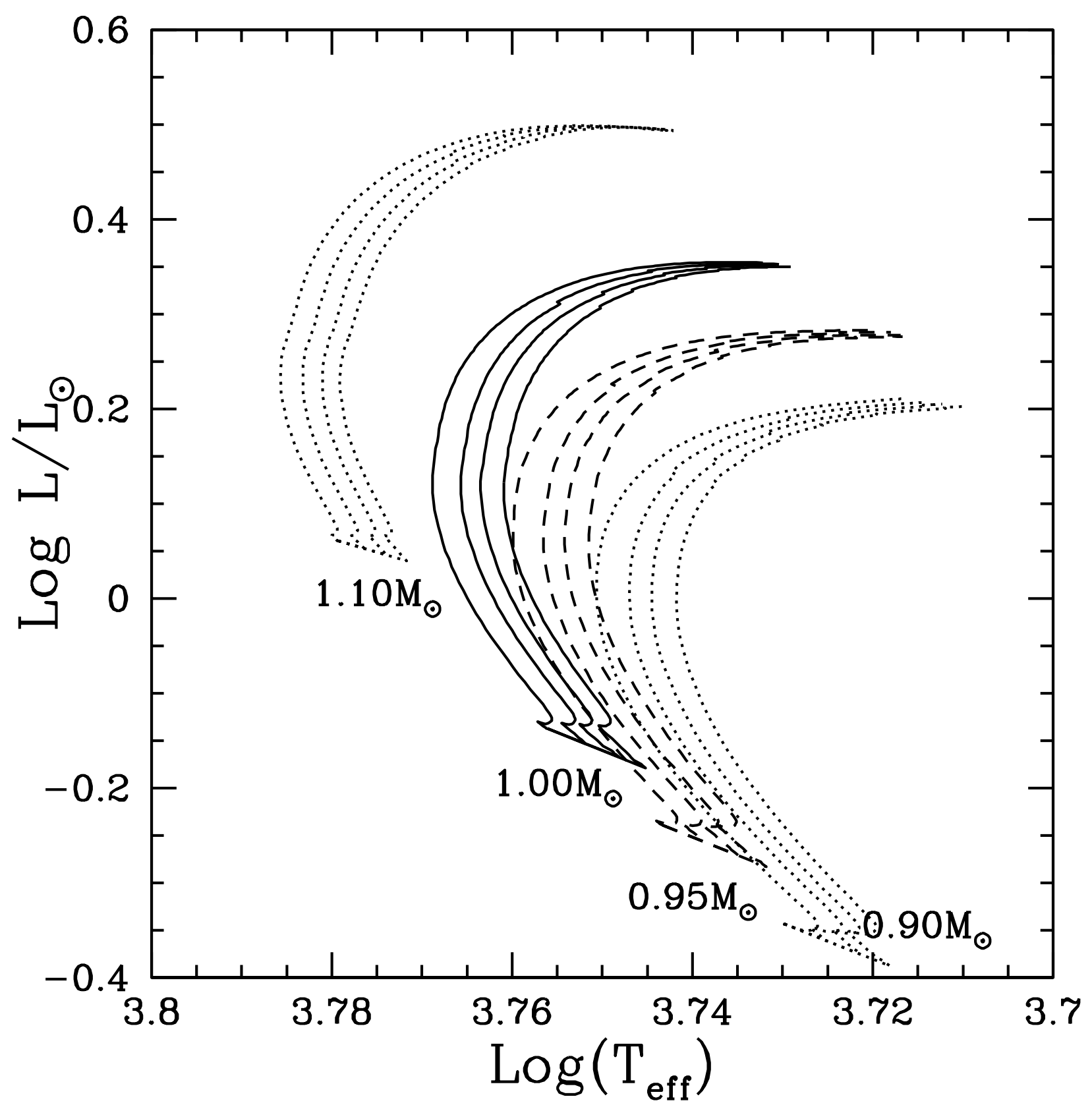

Fig. 5. - Polluted stellar evolution tracks for a fixed metal fraction of $\mathrm{Z}=0.02$, and various masses. Larger amounts of pollution $(\Delta \mathrm{Z})$ shift the tracks toward lower temperature. The values of $\Delta \mathrm{Z}$ included on the plot are $\Delta \mathrm{Z}=0.0,0.005,0.01$, and 0.015 for all masses. It is evident that the temperature shift due to pollution is roughly proportional to $\Delta \mathrm{Z}$. As the mass increases and the convection zone shrinks, this shift decreases, becoming nearly negligible for a $2.0 \mathrm{M}_{\odot}$ model. Ages range from 6.2-15.8 Gyr. 


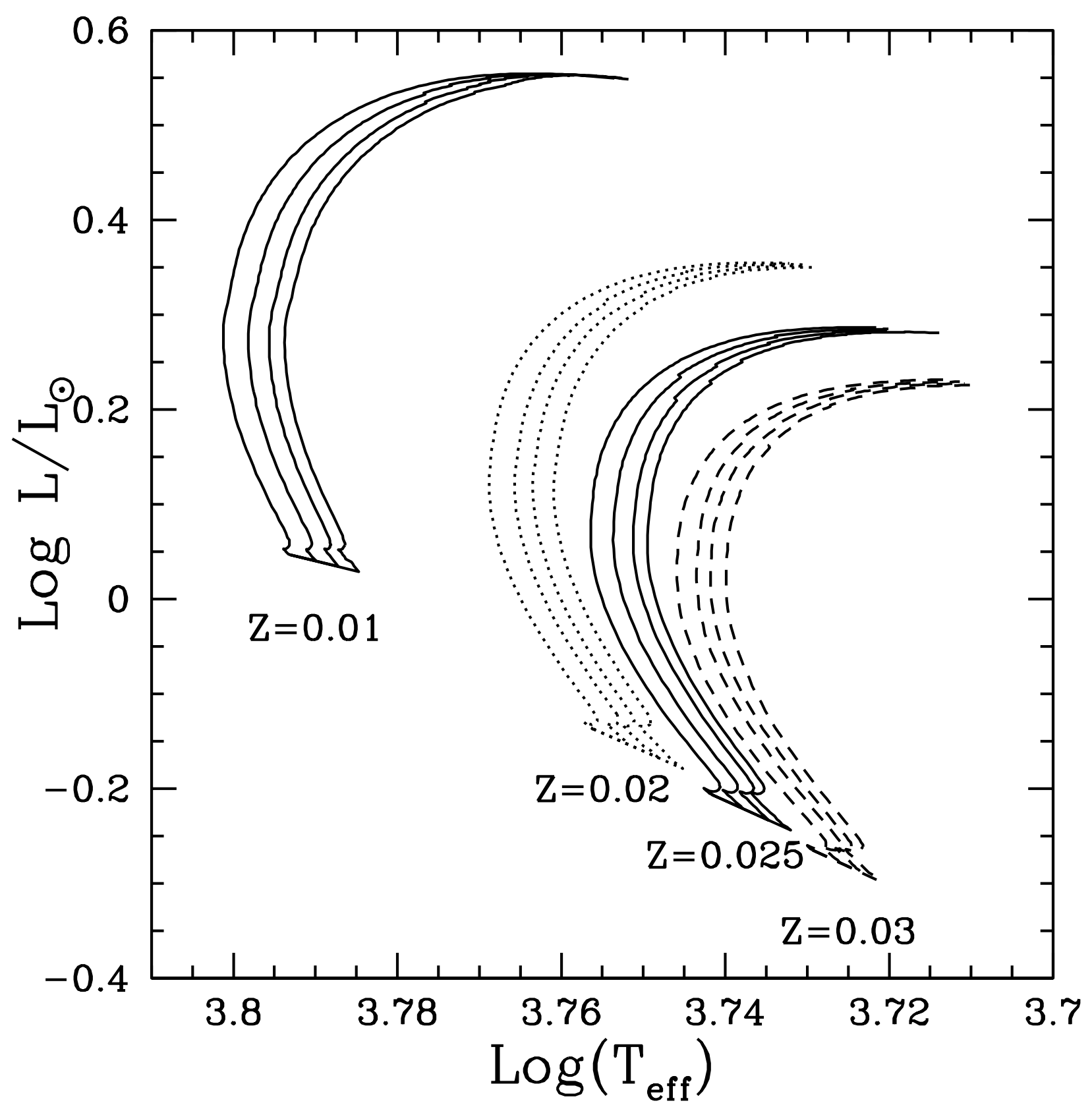

Fig. 6. - Polluted stellar evolution tracks for a $1 \mathrm{M}_{\odot}$ star, and several different metallicities. $\Delta \mathrm{Z}$ values included for each metallicity on the plot are $\Delta \mathrm{Z}=0.0,0.005,0.01$, and 0.015 . In all cases, temperature decreases with increasing $\Delta Z$. The locations of sets tracks are affected by metallicity changes, but the magnitudes of smaller shifts solely due to pollution show little variation. As with the fixed-metallicity models, the temperature shifts scale with amount of pollution. Tracks cover a range of approximately 7.2-14.4 Gyr. 


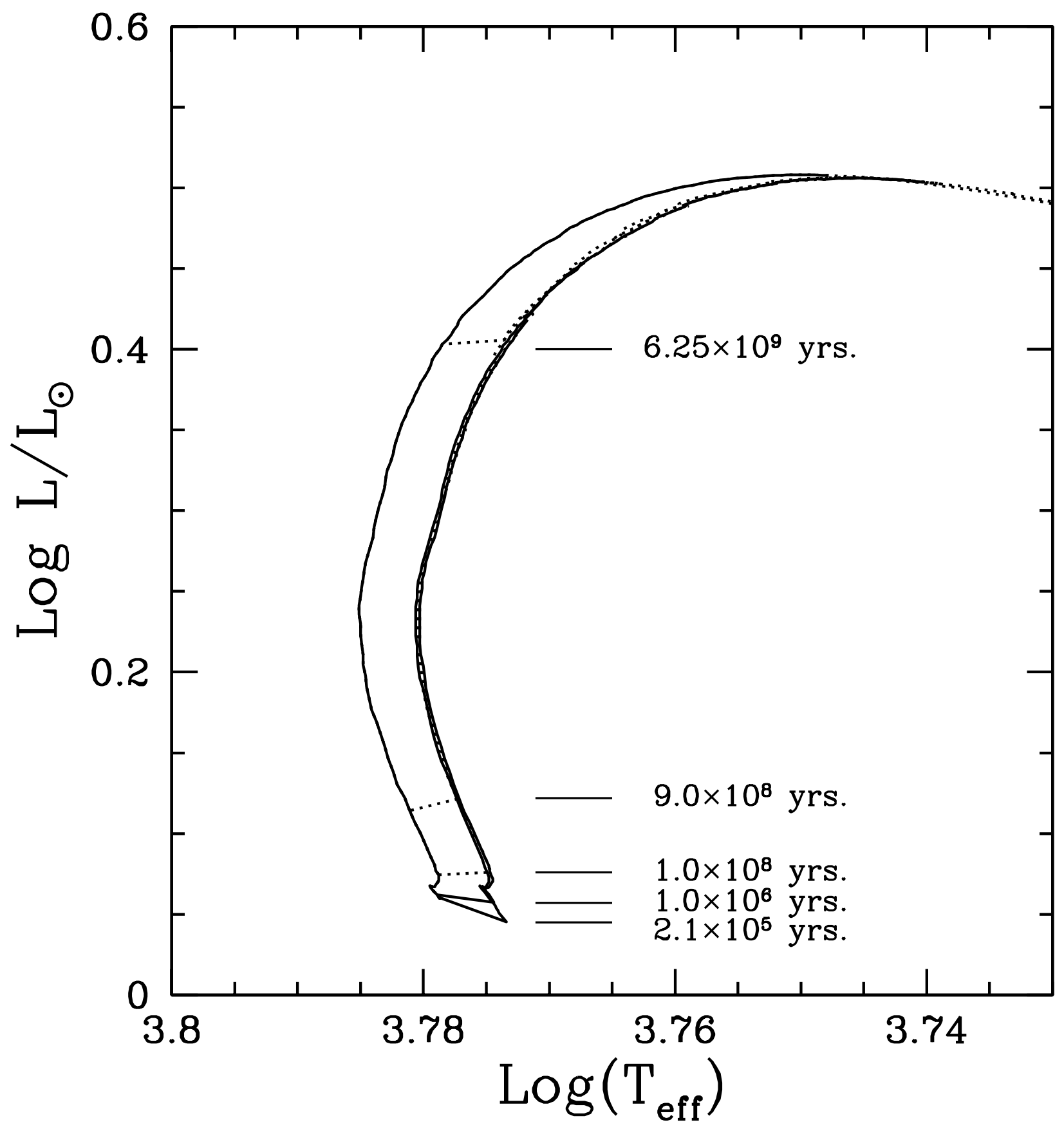

Fig. 7.- Results of varying the time of pollution in a $1.1 \mathrm{M}_{\odot}$ star with $\mathrm{Z}=0.02$ and $\Delta \mathrm{Z}=0.01$. We have calculated models for a variety of pollution times, and the resulting tracks are coincident, regardless of the age when the metallicity is increased (assuming that it is after the ZAMS time and before the subgiant stage). The plotted models follow the unpolluted track until pollution occurs, at which point they cross over to the polluted track (lower temperature). Age at crossover is noted on the plot. 


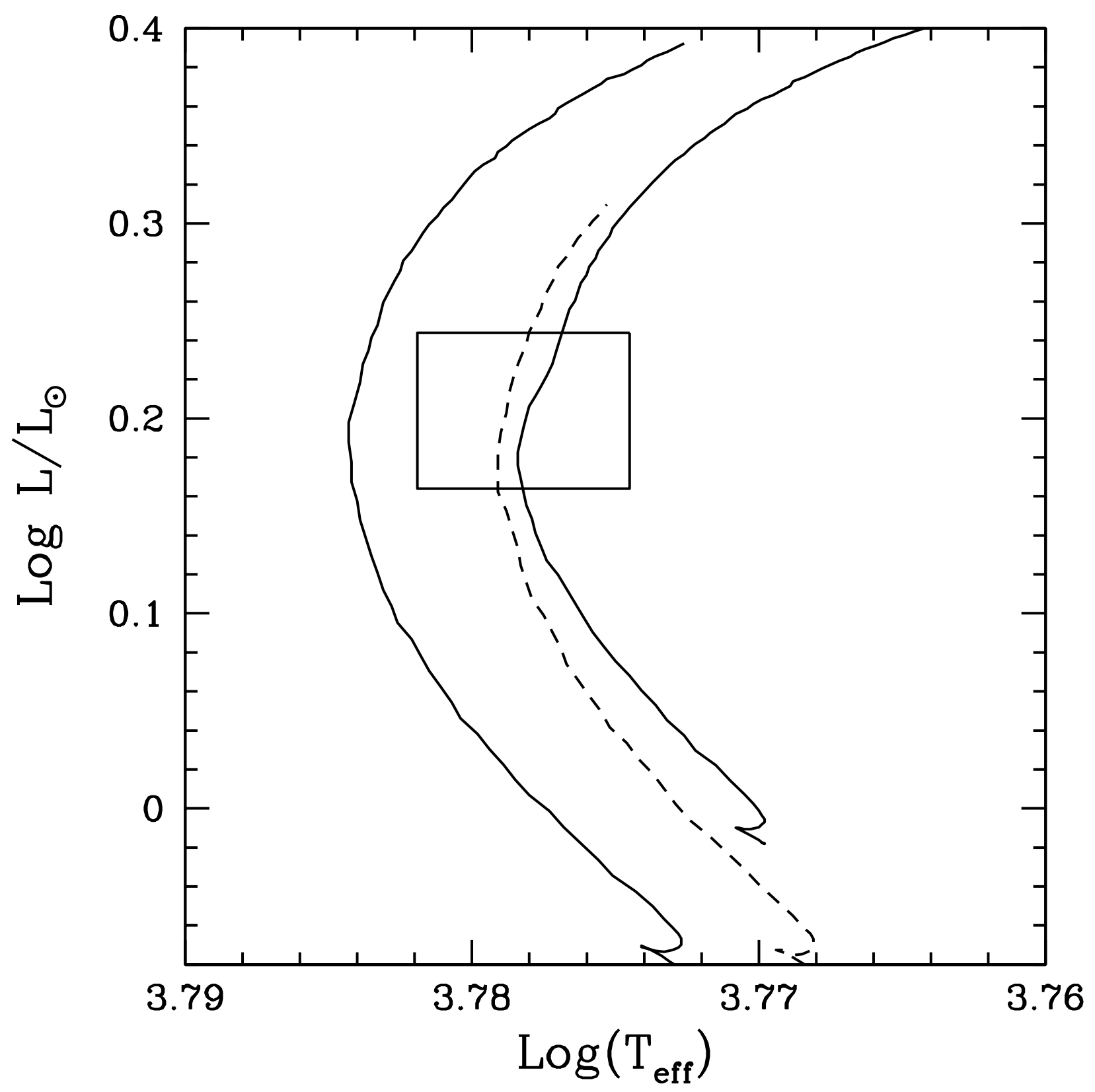

Fig. 8.- We have computed polluted and unpolluted evolutionary tracks for the star HD 209458, whose temperature-luminosity error box is shown in the center of the plot. A polluted stellar evolution track with parameters $\mathrm{M}=1.06 \mathrm{M}_{\odot}, \mathrm{Z}=0.019, \Delta \mathrm{Z}=0.001$, and $100 \%$ iron pollution is shown with a dot-dash pattern. The dashed curve illustrates the best-fit polluted track for HD 209458 when the accreted material is assumed to contain only metals in solar proportions; it is achieved with the values $\mathrm{M}=0.97 \mathrm{M}_{\odot}, \mathrm{Z}=0.013$, and $\Delta \mathrm{Z}=0.008$. The solid track within the box shows the best unpolluted evolutionary model for $\mathrm{Z}=0.02$, at $\mathrm{M}=1.06 \mathrm{M}_{\odot}$. For comparison, we have also plotted an unpolluted track of $\mathrm{M}=0.97 \mathrm{M}_{\odot}, \mathrm{Z}=0.013$, which appears to the left of the error box. 


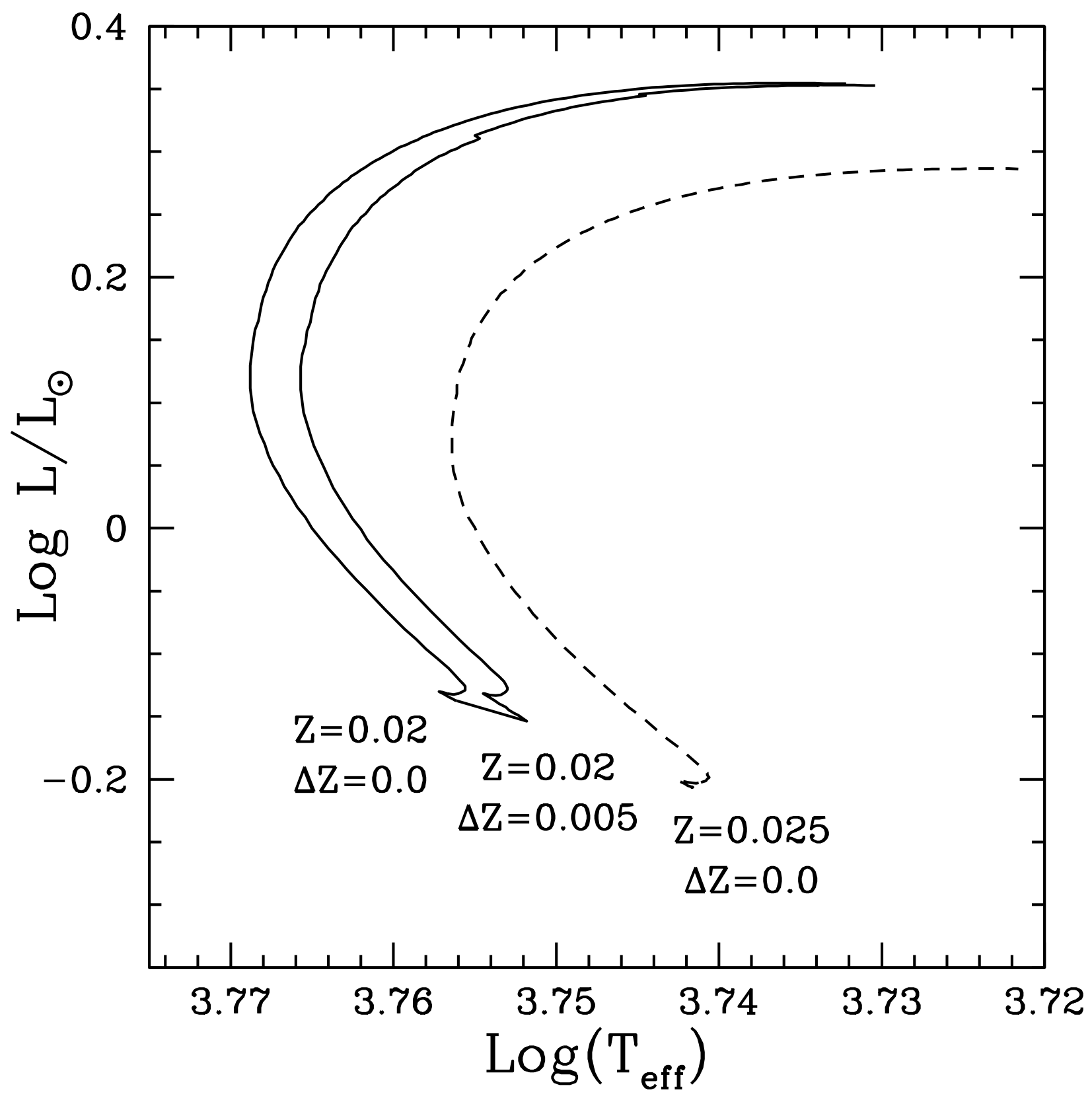

Fig. 9.- Three $1.0 \mathrm{M}_{\odot}$ tracks with $(\mathrm{Z}=0.02, \Delta \mathrm{Z}=0),(\mathrm{Z}=0.02, \Delta \mathrm{Z}=0.005)$, and $(\mathrm{Z}=0.025$, $\Delta Z=0$ ) illustrate the relation between models containing uniform $Z$ of 0.02 , uniform $Z$ of 0.025 , and interior $\mathrm{Z}$ of 0.02 /surface $\mathrm{Z}$ of 0.025 . Models with matching interior metallicities are much closer to each other than those with matching surface metallicities. 


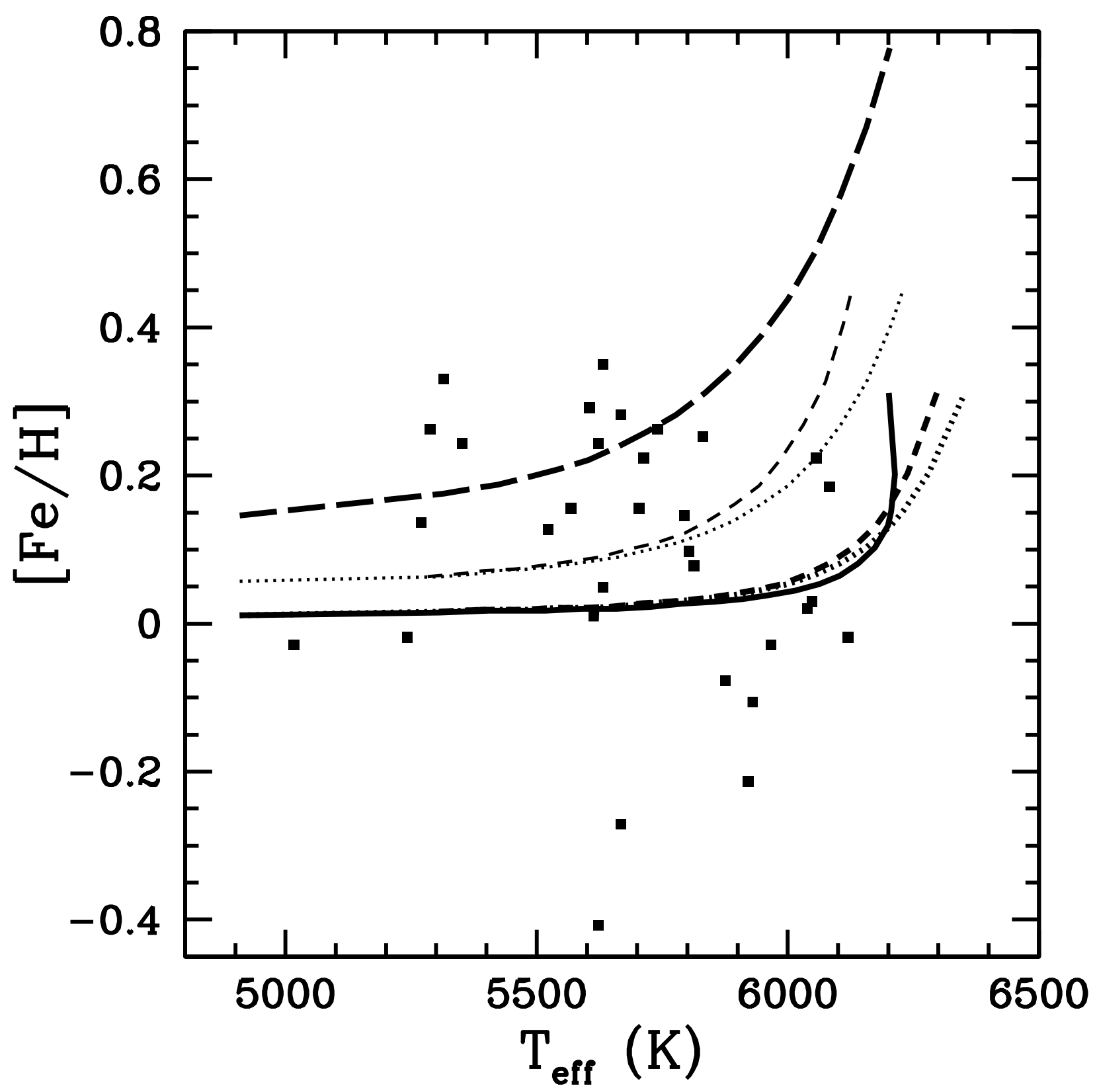

Fig. 10.- Amount of surface pollution as a function of stellar effective temperature. The latter correlates strongly with stellar mass (and hence - convection zone mass) on the main sequence. The points are nearby stars with known giant planets. The thin curves show metallicities expected for stars that accrete $40 \mathrm{M}_{\oplus}$ of pure metals (in solar proportions) on the ZAMS, while three lower thick curves depict metallicities calculated for stars that accrete $10 \mathrm{M}_{\oplus}$ of metal (also in solar proportionas). We have also included one model with $10 \mathrm{M}_{\oplus}$ of pure iron accretion (topmost curve). All models start with $Z_{\circ}=0.02$. We have plotted the metallicity trends for several different ages during the subsequent polluted evolution: 0.1 Myr (dotted lines and long dashes), 0.3 Myr (short dashes), and 3.0 Gyr (solid). Our models suppress the large enhancement at high temperatures seen in previous studies, but are still a poor fit to the observations. 